\title{
Colorimetric Analysis and Determination of Histamine in Samples of Yellowfin Tuna (Thunnus albacares) Marketed in Sardinia (Italy) by a Combination of Rapid Screening Methods and LC-MS/MS
}

\author{
Giovanni Luigi Pais ${ }^{1}$, Domenico Meloni ${ }^{1, *(\mathbb{D})}$, Alessandro Graziano Mudadu ${ }^{2}$, Luigi Crobu ${ }^{1}$, Alessandro Pulina ${ }^{2}$ \\ and Giannina Chessa ${ }^{2}$ \\ 1 Department of Veterinary Medicine, University of Sassari, Via Vienna 2, 07100 Sassari, Italy; \\ glpais@yahoo.it (G.L.P.); boesislac@hotmail.it (L.C.) \\ 2 Veterinary Public Health Institute of Sardinia, Via Duca degli Abruzzi 8, 07100 Sassari, Italy; \\ alessandro.mudadu@izs-sardegna.it (A.G.M.); alessandro.pulina@izs-sardegna.it (A.P.); \\ giannina.chessa@izs-sardegna.it (G.C.) \\ * Correspondence: dmeloni@uniss.it; Tel.: +39-079-229-570
}

check for updates

Citation: Pais, G.L.; Meloni, D. Mudadu, A.G.; Crobu, L.; Pulina, A.; Chessa, G. Colorimetric Analysis and Determination of Histamine in Samples of Yellowfin Tuna (Thunnus albacares) Marketed in Sardinia (Italy) by a Combination of Rapid Screening Methods and LC-MS/MS. Foods 2022, 11,639. https://doi.org/10.3390/ foods11050639

Academic Editors: Andrew G. Gehring, Maria Schirone and Pierina Visciano

Received: 9 December 2021 Accepted: 18 February 2022 Published: 22 February 2022

Publisher's Note: MDPI stays neutral with regard to jurisdictional claims in published maps and institutional affiliations.

Copyright: (C) 2022 by the authors. Licensee MDPI, Basel, Switzerland. This article is an open access article distributed under the terms and conditions of the Creative Commons Attribution (CC BY) license (https:// creativecommons.org/licenses/by/ $4.0 /)$

\begin{abstract}
The consumption of fishery products has been steadily increasing in recent decades. Among the quantitatively more important species, the yellowfin tuna (Thunnus albacares), is one of the main at-risk species as regards the possibility to present important levels of histamine and to be associated with the so-called "Scombroid Fish Poisoning". The main aim of the present study was to evaluate the colorimetric parameters, the occurrence, and the quantification of histamine contamination in yellowfin tuna samples marketed in Sardinia (Italy) by a combination of rapid screening and official control methods. A total of 20 samples of yellowfin tuna loins collected from large retailers, fishmongers and local markets were analyzed for the qualitative and quantitative determination of histamine by the lateral flow test HistaSure ${ }^{\mathrm{TM}}$ Fish Rapid Test and LC-MS/MS, respectively. Moreover, all the samples were examined to assess the conformity with the EU rules on labelling and subjected to colorimetric analysis according to the CIE- $L^{*} a^{*} b^{*}$ standard. Visual inspection of yellowfin tuna labels highlighted a $30 \%$ of non-compliances. A significant $(p<0.05)$ difference was reported for brightness $\left(L^{*}\right)$, redness $\left(a^{*}\right)$, and yellowness $\left(b^{*}\right)$. The results of histamine occurrence agreed with the food safety criteria (<100 mg/kg) laid down in EC Regulation 2073/2005 in the $95 \%$ and in the $90 \%$ of the samples with the rapid screening methods and LC-MS/MS, respectively. A highly significant sessional variation $(p<0.00001)$ was pointed out. Moreover, the two methods showed an agreement rate of $85 \%$. The results of the present study confirmed the utility of lateral flow tests for the fast qualitative determination of histamine in yellowfin tuna. Rapid screening test should be strengthened by comparison with the official method especially in case of uncertain or positive results.
\end{abstract}

Keywords: histamine; yellowfin tuna; food safety; screening; official control

\section{Introduction}

The consumption of fishery products has been steadily increasing in the European Union (EU) in recent decades: EU is the largest single market for imported fish and fishery products, representing $34 \%$ of total world imports [1]. The fishery products imported in Europe come from more than 120 countries worldwide and the EU puts high attention on quality, fishing, processing, and traceability along the supply chain [2-11]. Among the quantitatively more important species consumed in the EU, there is the yellowfin tuna (Thunnus albacares), a large pelagic fish that prevail in the tropics and subtropics [12]. In its natural state at the distribution stage, yellowfin tuna should be brown in colour. In the EU market, where it is forbidden to use chemicals to colour foods, yellowfin tuna available for 
sale in fish shops will look brown. One of the main sanitary frauds connected to the marketing of yellowfin tuna in the EU, is the fraudulent use of carbon monoxide (CO), where yellowfin tuna is coloured bright cherry red to look as everybody imagines fresh tuna to look. Since 2003, the presence of CO in yellowfin tuna from South-East Asian countries and from some Member States, has been repeatedly notified through the Rapid Alert System for Food and Feed (RASFF) [13]. In 2018, 79 people were arrested by Europol in “Operation Tarantello" for unauthorised treatment of tuna to promote a colour change [14]. CO is a colourless, odourless, and tasteless gas, toxic by inhalation but not by ingestion, injected into yellowfin tuna to counter the oxidation of the meat. $\mathrm{CO}$ reacts with the oxy-myoglobin to form Carboxymioglobin, a very stable and oxidation-resistant complex capable of giving muscle tissue a bright cherry red colour for several days. This very inviting colour it may not correspond to the real freshness of the fish and may mask bacterial spoilage. In histidine-rich fish such as yellowfin tuna, it may hide an excessive amount of histamine [13]. Yellowfin tuna is one of the main at-risk species in terms of presence of histamine at high levels: they present a very long shelf-life, are prepared with temperature variations that can support bacterial proliferation by Gram-(Morganella, Klebsiella, Proteus, E. coli and Hafnia) and metabolism of histidine-by-histidine decarboxylase decomposition [15]. High levels of histamine may be connected with the so-called "scombroid fish poisoning" [16,17], one of the most important seafood-borne disease worldwide [18,19]. In 2017, 105 cases of scombroid poisoning presented with mild episodes after consuming yellowfin tuna loins from Spain were reported in the EU, and out of these, 11 in Italy. Yellowfin tuna had been frozen and later thawed and sold as fresh vacuum-packed tuna [20]. Histamine was the only biological contaminant reported by the RASFF in the EU in 2020 [21]. Altogether, 14 alerts were associated to fish and products thereof mainly from Vietnam (n.6). In the EU, the EC Regulation 2073/2005 [22] reported the level of histamine in fishery products from fish species presenting high amounts of histidine (Scombridae, Clupeidae, Engraulidae, Coryfenidae, Pomatomidae, Scombresocidae) with acceptable quality ( $\leq 100 \mathrm{mg} / \mathrm{kg})$, marginal quality (between 100 and $200 \mathrm{mg} / \mathrm{kg}$ ) and inacceptable quality ( $\geq 200 \mathrm{mg} / \mathrm{kg}$ ). Due to the high-resolution power, sensitivity, flexibility, and reproducibility amongst all, the use of high-performance liquid chromatography (HPLC) is the analytical reference method in the EU $[23,24]$. Several alternative techniques have been previously described for the quantification of histamine [19,25-32] However, the high technical skills, cost and time required are the most important limits of these techniques. Therefore, sensitive, and rapid cost-effective methods to detect histamine have been developed [33] for Hazard Analysis Critical Control Points (HACCP) purposes in the fishery sector [34]. Commercial immunoassay tests for histamine qualitative determination are commonly accepted due to the ease of use and limited time requisites $[34,35]$. There is very little information about the histamine contamination in yellowfin tuna marketed in Italy, regarding the post-capture handling and marketing malpractices. Therefore, the main aim of the present study was to evaluate the respect of the food safety criteria for histamine provided by EC Regulation 2073/2005 [22] in yellowfin tuna samples marketed in Sardinia (Italy) by a combination of rapid screening and official control methods. The specific objectives were the following: (a) evaluation of the provisions of the Common Organisation of the Markets of Fishery and Aquaculture Products (CMO) in terms of labelling [36,37]; (b) colorimetric analysis according to the CIE- $L^{*} a^{*} b^{*}$ standard [38]; (c) qualitative determination of histamine by fast-track lateral flow tests (HistaSure ${ }^{\mathrm{TM}}$ Fish Rapid Test, LDN, Nordhorn, Germany); (d) quantitative determination of histamine by official control LC-MS/MS method; (e) comparison of the correspondence between the rapid screening methods and the official control methods.

\section{Materials and Methods}

\subsection{Collection of the Samples and Evaluation of European Union Labelling}

From October 2020 to February 2021, a total of 4 sampling visits (1-4) were scheduled in several market types (large retailers, fishmongers, and local fish markets) situated in the municipality of Sassari (Sardinia, Italy). A total of 20 samples (5 for each sampling 
day) of yellowfin tuna (T. albacares) loins were sampled randomly: in detail, 10 samples from large retailers, 5 samples from local fish markets and 5 samples from fish mongers. According to the availability of yellowfin tuna loins in the considered period, a few selling places were sampled several times. The label data of the yellowfin tuna samples were visually assessed to verify compliance with the rules of the Common Organization of the Markets in Fishery and Aquaculture Products (CMO) on the labelling and marketing of fish products [36,37]. All the samples were forwarded to the Veterinary Medicine Department at the University of Sassari (Italy) and were stored frozen before analysis. Samples were thawed in refrigerator to be subsequently subjected to colorimetric analysis, qualitative and quantitative determination of histamine.

\subsection{Colorimetric Analysis}

Colorimetric analysis was carried out with a digital Spectrophotometer Konica Minolta C508i (Konica Minolta Business Solutions Spa, Milan, Italy) according to the CIE $L^{*} a^{*} b^{*}$ system [38], standard illuminant D65, and $10^{\circ}$ standard observer specular component included [39]. Each tuna loin was assessed in triplicate and the mean value \pm standard deviation (s.d.) was used in statistic data elaboration.

\subsection{Qualitative Determination of Histamine}

Qualitative determination of histamine was carried out by the lateral flow test HistaSure ${ }^{\mathrm{TM}}$ Fish Rapid Test (LDN). To obtain results easily comparable with the food safety criteria laid down in EC Regulation 2073/2005 [22], the cut-off [40] has been set to $100 \mathrm{mg} / \mathrm{kg}$ histamine according to Crobu et al., 2021 [35]. Briefly, samples have been prepared for the test procedure according to the AOAC Official Method 937.07 [41]: $10 \mathrm{~g}$ of each tuna sample were added to $490 \mathrm{~mL}$ distilled water and homogenized for 1-2 min in a lab blender (Koenich, Munich, Germany). An aliquot of $100 \mu \mathrm{L}$ of the filtered homogenate was then pipetted into the Acylation Buffer Vials and incubated for $5 \mathrm{~min}$ at room temperature. Another aliquot of $100 \mu \mathrm{L}$ of the acylated samples were transferred into the Running Buffer Vials before adding the Lateral Flow Device and incubating for $5 \mathrm{~min}$. The Lateral Flow Device was then removed to visually read the results within $5 \mathrm{~min}$. A negative control represented by $100 \mu \mathrm{L}$ of distilled water was included in each sampling session. The results have been evaluated according to the HistaSure ${ }^{\mathrm{TM}}$ Fish Rapid Test (LDN) official protocol [40].

\subsection{Quantitative Determination of Histamine}

The quantification of histamine was carried out at the Laboratory of Environmental Chemistry and Toxicology of the Veterinary Public Health Institute of Sardinia in Sassari using a validated method LC-MS/MS fit for purpose requirements of EC Regulation 2073/2005 [22]. Briefly, $10.0 \mathrm{~g}$ of homogenized sample were weighed in $250 \mathrm{~mL}$ centrifuge containers and added to $190 \mathrm{~mL}$ of ultrapure water, stirred for $1 \mathrm{~min}$ and left to rest for $5 \mathrm{~min}$ (this step was repeated 3 times). Five $\mathrm{mL}$ of extract were withdrawn using a syringe and filtered through a Whatman glass microfiber filter (Maidstone, UK) inside a $10 \mathrm{~mL}$ tube. Subsequently, $100 \mu \mathrm{L}$ were transferred into a $5 \mathrm{~mL}$ volumetric flask, added with $50 \mu \mathrm{L}$ of Histamine D4 solution (Internal Standard) and adjusted to the desired volume with mobile phase A (Table 1). One $\mathrm{mL}$ of extract was filtered directly into an autosampler vial to proceed with instrumental analysis. The quantification of histamine was carried out through a UPLC Acquity I Class chromatography system coupled with Micromass Quattro Premier XE triple quadrupole mass spectrometer with electrospray ionization source (ESI) (Waters, Milford, CT, USA) by using an Acquity BEH HILIC $2.1 \mathrm{X}$ chromatographic column, $100 \mathrm{~mm}, 1.7 \mu \mathrm{m}$ (Waters) with a VanGuard BEH HILIC $1.7 \mu \mathrm{m}$ pre-column. Chromatography involved a gradient elution with the use of $10 \mathrm{mM}$ ammonium formate in 95:5 acetonitrile-water (mobile phase A) and $20 \mathrm{mM}$ ammonium formate ( $\mathrm{pH}$ 3) in ultra-pure water (mobile phase B), according to Table 1 . The mass analyzer operated in positive polarity with a capillary voltage set at $0.40 \mathrm{kV}$. Nitrogen was used as nebulization 
and desolvation gas at a flow of 50 and $900 \mathrm{~L} / \mathrm{h}$, respectively. The source temperature was maintained at $120{ }^{\circ} \mathrm{C}$, the solvation temperature at $350{ }^{\circ} \mathrm{C}$. The analysis was conducted in MRM (Multiple Reaction Monitoring) mode, using the transitions and acquisition parameters reported in Table 2. The method performances were established during validation studies. Parameters obtained are summarized in Table 3. The quality assurance and control of data were achieved using spiked materials and by checking that analysis complied the validation parameters method. Samples were analyzed in triplicate and the mean value \pm s.d. was used in statistic data elaboration.

Table 1. Gradient elution of the mobile phases A (acetonitrile-water) and B (ultra-pure water) with $10 \mathrm{mM}$ and $20 \mathrm{mM}$ ammonium formate.

\begin{tabular}{ccccc}
\hline $\begin{array}{c}\text { Time } \\
(\text { min) }\end{array}$ & \%A & \% B & $\begin{array}{c}\text { Flow } \\
(\mathbf{m L} / \mathbf{m i n})\end{array}$ & $\begin{array}{c}\text { Volume of Injection } \\
(\mu \mathrm{L})\end{array}$ \\
\hline 0.00 & 90 & 10 & & \\
0.10 & 90 & 10 & & \\
3.00 & 70 & 30 & 0.6 & \\
4.00 & 50 & 50 & & \\
4.50 & 50 & 50 & & \\
4.51 & 50 & 50 & & \\
\hline
\end{tabular}

Table 2. Transitions and acquisition parameters used in MRM (Multiple Reaction Monitoring) mode.

\begin{tabular}{cccccc}
\hline Analysis & $\begin{array}{c}\text { Parent } \\
(\mathbf{m} / \mathbf{z})\end{array}$ & $\begin{array}{c}\text { Daughter } \\
\mathbf{( m / \mathbf { z } )}\end{array}$ & Dwell Time (s) & $\begin{array}{c}\text { Cone } \\
\text { Voltage }(\mathbf{V})\end{array}$ & $\begin{array}{c}\text { Collision } \\
\text { Energy (V) }\end{array}$ \\
\hline Histamine & 111.9 & 67.9 & 0.025 & 50 & 22 \\
Histamine D4 & 111.9 & $94.9 \mathrm{Q}$ & 0.025 & 50 & 14 \\
\hline
\end{tabular}

Table 3. LC-MS/MS validation Parameters.

\begin{tabular}{cc}
\hline Analytical Parameters & Value \\
\hline Concentration Interval & $5-400 \mathrm{mg} / \mathrm{kg}$ \\
Linearity $\left(\mathrm{R}^{2}\right)$ & $>0.9995$ \\
Recoveries & $98-103 \%$ \\
Limit of quantification (LOQ) & $1.00 \mathrm{mg} / \mathrm{kg}$ \\
Intra-day precision (repeatability) & $0.7-3 \%$ \\
Inter-day precision (within-lab reproducibility) & $3-4 \%$ \\
\hline
\end{tabular}

\subsection{Statistical Analysis}

The differences in brightness $\left(L^{*}\right)$, redness $\left(a^{*}\right)$ and yellowness $\left(b^{*}\right)$ between the yellowfin tuna loins and in the concentration of histamine in relation to the sampling session and to the market type, were compared and analyzed with a one-way ANOVA [42]. Moreover, a multiple pairwise comparison between the means of groups through a Tukey HSD (honestly significant differences) post-hoc test was carried out [42]. The results were considered statistically significant when $p<0.05$.

\section{Results and Discussion}

\subsection{Collection of the Samples and Evaluation of European Union Labelling}

The visual inspection of the yellowfin tuna labels (Table 4) highlighted a high rate of non-compliances [36,37]. The 70\% of the labels showed T. albacares as a scientific denomination and yellowfin tuna as trade name. The production method and the category of the fishing gear were reported on $55 \%$ of the labels. Purse seine has been reported as the main fishing method. Yellowfin tuna caught with large-scale purse seines cannot immediately be handled after catching, with significant delay before cooling and subsequent freezing and storing $[43,44]$. In case of prolonged delays, post-mortal bacterial spoilage 
and accumulation of histamine is very frequent [45]. The fishing area (mainly Indian and Pacific Oceans) was indicated in 55\% of the labels. Only $40 \%$ of the labels indicated previous defrosting. According to previous studies [46,47], labelling was more accurate in large retailers than in local fish markets and fishmongers. In general, the smaller was the market type the more incomplete was the information [2]. The low rate of non-compliances found in large retailers should be linked to the accuracy in the information transmission along this marketing circuit than the other market types [2], to the applied procedures for the referencing of the suppliers and to the specific training programs of the market staff in charge [48]. As previously reported, not detailed labelling or mislabeling may cause consumers' misunderstanding [2,11,46,47,49-54].

\subsection{Colorimetric Analysis}

The results of the colorimetric analysis performed on the yellowfin tuna samples are shown in Table 5. The average values ( \pm s.d.) of brightness $\left(L^{*}\right)$, redness $\left(a^{*}\right)$, and yellowness $\left(b^{*}\right)$ calculated according to the reference standard CIE- $L * a * b *$ [38], were the following: $L^{*}=58.90 \pm 3.20 ; a^{*}=8.67 \pm 1.79 ; b^{*}=8.38 \pm 1.44$. A significant $(p<0.05)$ difference was reported for the colorimetric parameters (Table 6).

\subsection{Qualitative Determination of Histamine}

The qualitative determination of histamine performed by HistaSure ${ }^{\mathrm{TM}}$ Fish Rapid Test (LDN) highlighted that $95 \%$ of the samples were always $<100 \mathrm{mg} / \mathrm{kg}$ and agreed with the EU food safety criteria [22]. Only one sample (2C) showed levels $>100 \mathrm{mg} / \mathrm{kg}$ (Table 7).

\subsection{Quantitative Determination of Histamine}

The quantitative determination of histamine carried out using the official LC-MS/MS method showed that $90 \%$ of the samples were $<100 \mathrm{mg} / \mathrm{kg}$ in accordance with the EU food safety criteria [22]. Two samples (1B and 1E) showed levels $>100 \mathrm{mg} / \mathrm{kg}$ (Table 7). ANOVA and Tukey test showed a highly significant sessional variation $(p<0.00001)$ of histamine concentration in examined yellowfin tuna samples (Table 8). Previous studies carried out in tuna samples collected at the retail stage showed histamine levels of $0.45-83.73 \mathrm{mg} / \mathrm{kg}$ [18]. Histamine was found at mean levels of $8.9 \mathrm{mg} / \mathrm{kg}$ in $83.3 \%$ of tuna samples in Spain, while a sample collected from the Netherlands showed high histamine levels $(1439 \mathrm{mg} / \mathrm{kg})$ exceeding the EU food safety criteria [18,55-57]. 
Table 4. Labelling of yellowfin tuna samples included in the study.

\begin{tabular}{|c|c|c|c|c|c|c|c|c|c|c|c|c|c|c|c|c|c|c|c|c|c|}
\hline \multirow{2}{*}{$\begin{array}{l}\text { Mandatory } \\
\text { Information }\end{array}$} & \multicolumn{20}{|c|}{ Samples } & \multirow{2}{*}{ Total (\%) } \\
\hline & $1 \mathrm{~A}$ & 1B & $1 \mathrm{C}$ & 1D & $1 \mathrm{E}$ & $2 \mathrm{~A}$ & 2B & $2 \mathrm{C}$ & $2 \mathrm{D}$ & $2 \mathrm{E}$ & $3 \mathrm{~A}$ & $3 B$ & $3 C$ & 3D & $3 \mathrm{E}$ & $4 \mathrm{~A}$ & $4 B$ & $4 C$ & $4 \mathrm{D}$ & $4 \mathrm{E}$ & \\
\hline Market type & $\begin{array}{l}\text { Local } \\
\text { fish } \\
\text { mar- } \\
\text { ket }\end{array}$ & $\begin{array}{l}\text { Local } \\
\text { fish } \\
\text { mar- } \\
\text { ket }\end{array}$ & $\begin{array}{l}\text { Fish- } \\
\text { monger }\end{array}$ & $\begin{array}{l}\text { Fish- } \\
\text { monger }\end{array}$ & $\begin{array}{l}\text { Fish- } \\
\text { monger }\end{array}$ & $\begin{array}{l}\text { Large } \\
\text { Re-- } \\
\text { tailer }\end{array}$ & $\begin{array}{l}\text { Local } \\
\text { fish } \\
\text { mar- } \\
\text { ket }\end{array}$ & $\begin{array}{l}\text { Fish- } \\
\text { monger }\end{array}$ & $\begin{array}{l}\text { Large } \\
\text { Re- } \\
\text { tailer }\end{array}$ & $\begin{array}{l}\text { Large } \\
\text { Re- } \\
\text { tailer }\end{array}$ & $\begin{array}{l}\text { Large } \\
\text { Re-- } \\
\text { tailer }\end{array}$ & $\begin{array}{l}\text { Local } \\
\text { fish } \\
\text { mar- } \\
\text { ket }\end{array}$ & $\begin{array}{l}\text { Large } \\
\text { Re-- } \\
\text { tailer }\end{array}$ & $\begin{array}{l}\text { Large } \\
\text { Re-- } \\
\text { tailer }\end{array}$ & $\begin{array}{l}\text { Large } \\
\text { Re-- } \\
\text { tailer }\end{array}$ & $\begin{array}{l}\text { Large } \\
\text { Re- } \\
\text { tailer }\end{array}$ & $\begin{array}{l}\text { Local } \\
\text { fish } \\
\text { mar- } \\
\text { ket }\end{array}$ & $\begin{array}{l}\text { Fish- } \\
\text { monger }\end{array}$ & $\begin{array}{l}\text { Large } \\
\text { Re- } \\
\text { tailer }\end{array}$ & $\begin{array}{l}\text { Large } \\
\text { Re- } \\
\text { tailer }\end{array}$ & \\
\hline $\begin{array}{l}\text { Scientific name } \\
\text { (Thunnus } \\
\text { albacares) }\end{array}$ & yes & yes & - & - & - & yes & yes & - & yes & yes & yes & yes & yes & yes & yes & - & - & yes & yes & Yes & 70 \\
\hline $\begin{array}{l}\text { Commercial } \\
\text { designation } \\
\text { (Yellowfin } \\
\text { tuna) }\end{array}$ & yes & yes & - & - & - & yes & yes & - & yes & yes & yes & yes & yes & yes & yes & - & - & yes & yes & yes & 70 \\
\hline $\begin{array}{l}\text { Production } \\
\text { method }\end{array}$ & - & - & - & - & - & yes & - & & yes & yes & yes & yes & yes & yes & yes & - & - & yes & yes & yes & 55 \\
\hline Fishing area & - & - & - & - & - & yes & - & - & yes & yes & yes & yes & yes & yes & yes & - & - & yes & yes & yes & 55 \\
\hline Fishing gear & - & - & - & - & - & yes & - & - & yes & yes & yes & yes & yes & yes & yes & - & - & yes & yes & yes & 55 \\
\hline Defrosting & - & - & - & - & - & yes & - & - & yes & yes & yes & - & - & yes & yes & - & - & - & yes & yes & 40 \\
\hline
\end{tabular}

Table 5. Colorimetric parameters (mean \pm s. d.) of yellowfin tuna samples included in the study.

\begin{tabular}{|c|c|c|c|c|c|c|c|c|c|c|c|c|c|c|c|c|c|c|c|c|c|}
\hline \multirow{2}{*}{$\begin{array}{l}\text { Colorimetric } \\
\text { Parameters }\end{array}$} & \multicolumn{20}{|c|}{ Samples (Mean* \pm s.d.) } & \multirow[t]{2}{*}{$\begin{array}{c}\text { Mean Values } \\
\pm \text { s.d. }\end{array}$} \\
\hline & 1A & 1B & $1 C$ & 1D & 1E & $2 \mathrm{~A}$ & 2B & $2 \mathrm{C}$ & $2 \mathrm{D}$ & $2 \mathrm{E}$ & $3 \mathrm{~A}$ & 3B & $3 C$ & 3D & $3 \mathrm{E}$ & $4 \mathrm{~A}$ & $4 B$ & $4 \mathrm{C}$ & $4 \mathrm{D}$ & $4 \mathrm{E}$ & \\
\hline & 59.53 & 58.57 & 61.56 & 51.42 & 55.17 & 61 & 48.77 & 52.55 & 59.79 & 62.15 & 65.95 & 57.47 & 65.02 & 67.5 & 62.55 & 54.23 & 62.99 & 57.43 & 57.99 & 56.48 & \\
\hline $\begin{array}{c}\left(L^{*}\right) \\
\text { brightness }\end{array}$ & $\begin{array}{c} \pm \\
1.71\end{array}$ & $\begin{array}{c} \pm \\
0.47\end{array}$ & $\begin{array}{c} \pm \\
4.29\end{array}$ & $\begin{array}{c} \pm \\
3.50\end{array}$ & $\begin{array}{c} \pm \\
3.67\end{array}$ & $\begin{array}{c} \pm \\
3.28\end{array}$ & $\begin{array}{c} \pm \\
5.96\end{array}$ & $\begin{array}{c} \pm \\
3.52\end{array}$ & $\begin{array}{c} \pm \\
2.03\end{array}$ & $\begin{array}{c} \pm \\
4.97\end{array}$ & $\begin{array}{c} \pm \\
3.38\end{array}$ & $\begin{array}{c} \pm \\
6.52\end{array}$ & $\begin{array}{c} \pm \\
1.31\end{array}$ & $\begin{array}{c} \pm \\
0.70\end{array}$ & $\begin{array}{c} \pm \\
1.24\end{array}$ & $\begin{array}{c} \pm \\
3.90\end{array}$ & $\begin{array}{c} \pm \\
3.91\end{array}$ & $\begin{array}{c} \pm \\
1.65\end{array}$ & $\begin{array}{c} \pm \\
2.50\end{array}$ & $\begin{array}{c} \pm \\
5.57\end{array}$ & $58.90 \pm 3.20$ \\
\hline & 7.52 & 11.97 & 9.55 & 10.33 & 4.15 & 4.65 & 12.79 & 13.13 & 3.80 & 7.60 & 6.88 & 9.23 & 10.22 & 7.73 & 4.50 & 8.63 & 13.76 & 11.04 & 8.87 & 7.03 & \\
\hline redness & $\begin{array}{c} \pm \\
0.85\end{array}$ & $\stackrel{ \pm}{2.57}$ & $\begin{array}{c} \pm \\
0.53\end{array}$ & $\begin{array}{l} \pm \\
1.70\end{array}$ & $\begin{array}{c} \pm \\
0.23\end{array}$ & $\begin{array}{c} \pm \\
0.53\end{array}$ & $\begin{array}{c} \pm \\
1.21\end{array}$ & $\begin{array}{c} \pm \\
4.90\end{array}$ & $\begin{array}{c} \pm \\
1.24\end{array}$ & $\begin{array}{c} \pm \\
2.63\end{array}$ & $\begin{array}{c} \pm \\
0.53\end{array}$ & $\begin{array}{c} \pm \\
3.75\end{array}$ & $\begin{array}{c} \pm \\
1.60\end{array}$ & $\begin{array}{c} \pm \\
1.02\end{array}$ & $\stackrel{ \pm}{ \pm .38}$ & $\begin{array}{c} \pm \\
1.99\end{array}$ & $\stackrel{ \pm}{ \pm .44}$ & $\begin{array}{c} \pm \\
2.78\end{array}$ & $\begin{array}{c} \pm \\
1.94\end{array}$ & $\begin{array}{c} \pm \\
2.08\end{array}$ & $8.67 \pm 1.79$ \\
\hline & 5.42 & 9.37 & 8.36 & 7.87 & 9.24 & 8.33 & 13.17 & 10.38 & 7.71 & 8.56 & 6.73 & 5.76 & 8.59 & 6.04 & 6.77 & 12.31 & 5.82 & 6.96 & 9.83 & 10.47 & \\
\hline $\begin{array}{c}\left(b^{*}\right) \\
\text { yellowness }\end{array}$ & $\begin{array}{c} \pm \\
0.63\end{array}$ & $\begin{array}{c} \pm \\
1.99\end{array}$ & $\begin{array}{c} \pm \\
1.04\end{array}$ & $\stackrel{ \pm}{2.11}$ & $\begin{array}{c} \pm \\
1.54\end{array}$ & $\begin{array}{c} \pm \\
0.19\end{array}$ & $\begin{array}{c} \pm \\
1.96\end{array}$ & $\begin{array}{c} \pm \\
3.47\end{array}$ & $\stackrel{ \pm}{ \pm .55}$ & $\begin{array}{c} \pm \\
1.96\end{array}$ & $\begin{array}{c} \pm \\
0.84\end{array}$ & $\begin{array}{c} \pm \\
2.70\end{array}$ & $\stackrel{ \pm}{1.15}$ & $\begin{array}{c} \pm \\
1.08\end{array}$ & $\stackrel{ \pm}{ \pm .58}$ & $\stackrel{ \pm}{ \pm .27}$ & $\stackrel{ \pm}{ \pm}$ & $\begin{array}{c} \pm \\
1.61\end{array}$ & $\begin{array}{c} \pm \\
0.65\end{array}$ & $\begin{array}{c} \pm \\
2.09\end{array}$ & $8.38 \pm 1.44$ \\
\hline
\end{tabular}


Table 6. Differences among the colorimetric parameters in yellowfin tuna samples from ANOVA and the Tukey test.

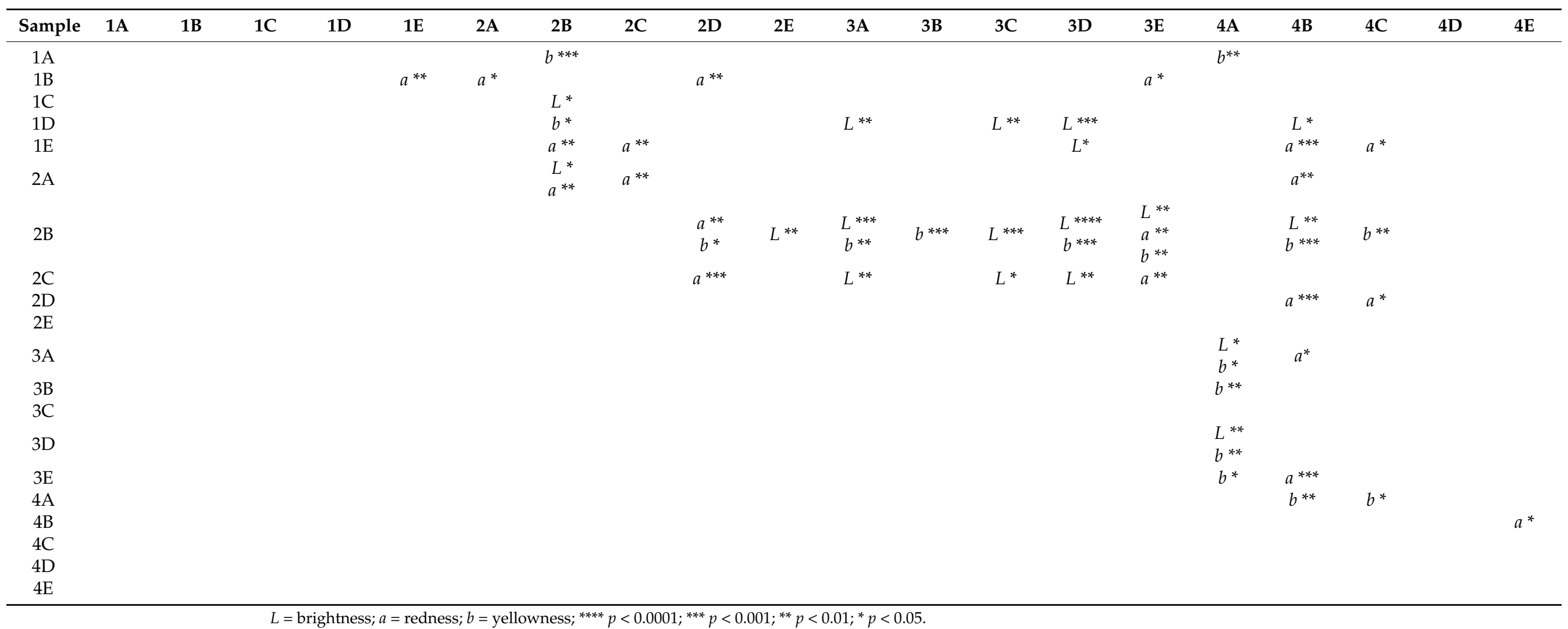


Table 7. Qualitative and quantitative determination of histamine in of yellowfin tuna samples included in the study.

\begin{tabular}{|c|c|c|c|c|c|c|c|c|c|c|c|c|c|c|c|c|c|c|c|c|}
\hline \multicolumn{21}{|c|}{ Samples (Mean \pm s.d.) } \\
\hline Market Type & $\begin{array}{c}\text { Local } \\
\text { Fish } \\
\text { Mar- } \\
\text { ket }\end{array}$ & $\begin{array}{c}\text { Local } \\
\text { Fish } \\
\text { Mar- } \\
\text { ket }\end{array}$ & $\begin{array}{l}\text { Fish- } \\
\text { monger }\end{array}$ & $\begin{array}{l}\text { Fish- } \\
\text { monger }\end{array}$ & $\begin{array}{l}\text { Fish- } \\
\text { monger }\end{array}$ & $\begin{array}{c}\text { Large } \\
\text { Re- } \\
\text { tailer }\end{array}$ & $\begin{array}{c}\text { Local } \\
\text { Fish } \\
\text { Mar- } \\
\text { ket }\end{array}$ & $\begin{array}{l}\text { Fish- } \\
\text { monger }\end{array}$ & $\begin{array}{c}\text { Large } \\
\text { Re- } \\
\text { tailer }\end{array}$ & $\begin{array}{c}\text { Large } \\
\text { Re- } \\
\text { tailer }\end{array}$ & $\begin{array}{c}\text { Large } \\
\text { Re- } \\
\text { tailer }\end{array}$ & $\begin{array}{c}\text { Local } \\
\text { Fish } \\
\text { Mar- } \\
\text { ket }\end{array}$ & $\begin{array}{c}\text { Large } \\
\text { Re- } \\
\text { tailer }\end{array}$ & $\begin{array}{c}\text { Large } \\
\text { Re- } \\
\text { tailer }\end{array}$ & $\begin{array}{c}\text { Large } \\
\text { Re- } \\
\text { tailer }\end{array}$ & $\begin{array}{c}\text { Large } \\
\text { Re- } \\
\text { tailer }\end{array}$ & $\begin{array}{c}\text { Local } \\
\text { Fish } \\
\text { Mar- } \\
\text { ket }\end{array}$ & $\begin{array}{l}\text { Fish- } \\
\text { monger }\end{array}$ & $\begin{array}{c}\text { Large } \\
\text { Re- } \\
\text { tailer }\end{array}$ & $\begin{array}{c}\text { Large } \\
\text { Re- } \\
\text { tailer }\end{array}$ \\
\hline $\begin{array}{l}\text { Histamine de- } \\
\text { termination } \\
\text { Qualitative }\end{array}$ & $1 \mathrm{~A}$ & 1B & $1 \mathrm{C}$ & $1 \mathrm{D}$ & $1 \mathrm{E}$ & $2 \mathrm{~A}$ & $2 B$ & $2 \mathrm{C}$ & $2 \mathrm{D}$ & $2 \mathrm{E}$ & $3 \mathrm{~A}$ & 3B & $3 \mathrm{C}$ & $3 \mathrm{D}$ & $3 \mathrm{E}$ & $4 \mathrm{~A}$ & $4 \mathrm{~B}$ & $4 \mathrm{C}$ & $4 \mathrm{D}$ & $4 \mathrm{E}$ \\
\hline $\begin{array}{l}\text { (HistaSure TM } \\
\text { Fish Rapid } \\
\text { Test) }\end{array}$ & $\begin{array}{l}<100 \\
\mathrm{mg} / \mathrm{kg}\end{array}$ & $\begin{array}{l}<100 \\
\mathrm{mg} / \mathrm{kg}\end{array}$ & $\begin{array}{l}<100 \\
\mathrm{mg} / \mathrm{kg}\end{array}$ & $\begin{array}{l}<100 \\
\mathrm{mg} / \mathrm{kg}\end{array}$ & $\begin{array}{l}<100 \\
\mathrm{mg} / \mathrm{kg}\end{array}$ & $\begin{array}{l}<100 \\
\mathrm{mg} / \mathrm{kg}\end{array}$ & $\begin{array}{l}<100 \\
\mathrm{mg} / \mathrm{kg}\end{array}$ & $\begin{array}{l}>100 \\
\mathrm{mg} / \mathrm{kg}\end{array}$ & $\begin{array}{l}<100 \\
\mathrm{mg} / \mathrm{kg}\end{array}$ & $\begin{array}{l}<100 \\
\mathrm{mg} / \mathrm{kg}\end{array}$ & $\begin{array}{l}<100 \\
\mathrm{mg} / \mathrm{kg}\end{array}$ & $\begin{array}{l}<100 \\
\mathrm{mg} / \mathrm{kg}\end{array}$ & $\begin{array}{l}<100 \\
\mathrm{mg} / \mathrm{kg}\end{array}$ & $\begin{array}{l}<100 \\
\mathrm{mg} / \mathrm{kg}\end{array}$ & $\begin{array}{l}<100 \\
\mathrm{mg} / \mathrm{kg}\end{array}$ & $\begin{array}{l}<100 \\
\mathrm{mg} / \mathrm{kg}\end{array}$ & $\begin{array}{l}<100 \\
\mathrm{mg} / \mathrm{kg}\end{array}$ & $\begin{array}{l}<100 \\
\mathrm{mg} / \mathrm{kg}\end{array}$ & $\begin{array}{l}<100 \\
\mathrm{mg} / \mathrm{kg}\end{array}$ & $\begin{array}{l}<100 \\
\mathrm{mg} / \mathrm{kg}\end{array}$ \\
\hline
\end{tabular}

Table 8. Differences among the concentration of histamine in yellowfin tuna samples from ANOVA and the Tukey test.

\begin{tabular}{|c|c|c|c|c|c|c|c|c|c|c|c|c|c|c|c|c|c|c|c|c|}
\hline Sample & 1A & 1B & $1 \mathrm{C}$ & 1D & $1 \mathrm{E}$ & $2 \mathrm{~A}$ & 2B & $2 \mathrm{C}$ & 2D & $2 \mathrm{E}$ & $3 \mathbf{A}$ & 3B & $3 C$ & 3D & $3 E$ & $4 \mathrm{~A}$ & 4B & $4 \mathrm{C}$ & 4D & $4 \mathrm{E}$ \\
\hline $1 \mathrm{~A}$ & & $* * * * *$ & $* * * * * *$ & $* * * * * *$ & $* * * * * *$ & $* * * * * *$ & $* * * * * *$ & $* * * * * *$ & $* * * * *$ & $* * * * *$ & $* * * * *$ & $* * * * * *$ & $* * * * *$ & $* * * * *$ & $* * * * *$ & $* * * * * *$ & $* * * * * *$ & $* * * * *$ & $* * * * *$ & $* * * * * *$ \\
\hline $1 \mathrm{~B}$ & & & $* * * * *$ & $* * * * *$ & $* * * * * *$ & $* * * * * *$ & $* * * * *$ & $* * * * *$ & $* * * * *$ & $* * * * *$ & $* * * * *$ & $* * * * *$ & $* * * * *$ & $* * * * *$ & $* * * * *$ & $* * * * *$ & $* * * * * *$ & $* * * * *$ & $* * * * *$ & $* * * * *$ \\
\hline $1 \mathrm{C}$ & & & & $* * *$ & $* * * * *$ & $* * * * *$ & $* * * * *$ & $* * * * *$ & $* * * * *$ & $* * * * *$ & $* * * * *$ & $*$ & $* * * * *$ & $* * * * *$ & $* * * * *$ & $* * * * * *$ & $* * * * * *$ & $* * * * * *$ & $* * * * *$ & $* * * * *$ \\
\hline 1D & & & & & $* * * * * *$ & $* * * * *$ & $* * * * *$ & $* * * * *$ & $* * * * *$ & $* * * * *$ & $* * * * *$ & & $* * * * *$ & $* * * * *$ & $* * * * *$ & $* * * * *$ & $* * * * *$ & $* * * * *$ & $* * * * *$ & $* * * * * *$ \\
\hline $1 \mathrm{E}$ & & & & & & $* * * * * *$ & $* * * * *$ & $* * * * *$ & $* * * * *$ & $* * * * *$ & $* * * * *$ & $* * * * *$ & $* * * * *$ & $* * * * *$ & $* * * * *$ & $* * * * *$ & $* * * * *$ & $* * * * *$ & $* * * * *$ & $* * * * * *$ \\
\hline $2 \mathrm{~A}$ & & & & & & & $* * * * * *$ & $* * * * * *$ & $* * * * * *$ & $* * * * *$ & $* * * * * *$ & $* * * * * *$ & $* * * * * *$ & $* * * * *$ & $* * * * *$ & $* * * * * *$ & $* * * * * *$ & $* * * * * *$ & $* * * * * *$ & $* * * * * *$ \\
\hline $2 B$ & & & & & & & & $* * * * * *$ & $* * * * * *$ & $* * * * *$ & $* * * * * *$ & $* * * * * *$ & $* * * * * *$ & $* * * * *$ & $* * * * *$ & $* * * * * *$ & & $* * * * * *$ & $* * * * * *$ & $* * * * * *$ \\
\hline $2 \mathrm{C}$ & & & & & & & & & $* * * * * *$ & $* * * * *$ & $* * * * * *$ & $* * * * * *$ & $* * * * * *$ & $* * * * *$ & $* * * * *$ & $* * * * * *$ & $* * * * * *$ & $* * * * * *$ & $* * * * * *$ & $* * * * * *$ \\
\hline $2 \mathrm{D}$ & & & & & & & & & & $* * *$ & $* * * * * *$ & $* * * * * *$ & $* * * * * *$ & $* * * * *$ & $* * * * *$ & $* * * * *$ & $* * * * * *$ & $* * * * * *$ & $* * * * * *$ & $* * * * * *$ \\
\hline $2 \mathrm{E}$ & & & & & & & & & & & $* * * * * *$ & $* * * * * *$ & $* * * * * *$ & & $* * * * *$ & $* *$ & $* * * * * *$ & $* * * * * *$ & $* * * * * *$ & $* * * * * *$ \\
\hline $3 \mathrm{~A}$ & & & & & & & & & & & & $* * * * * *$ & $* * * * * *$ & * & $* * * * *$ & & $* * * * * *$ & & $* * * * * *$ & $* * * * * *$ \\
\hline $3 \mathrm{~B}$ & & & & & & & & & & & & & $* * * * * *$ & $* * * * *$ & $* * * * *$ & $* * * * * *$ & $* * * * * *$ & $* * * * * *$ & $* * * * * *$ & $* * * * * *$ \\
\hline $3 \mathrm{C}$ & & & & & & & & & & & & & & $* * * * *$ & $* * * * *$ & $* * * * *$ & $* * * * *$ & $* * * * *$ & $* * * * *$ & $* * * * *$ \\
\hline $3 \mathrm{D}$ & & & & & & & & & & & & & & & $* * * * *$ & & $* * * * *$ & * & $* * * * *$ & $* * * * * *$ \\
\hline $3 \mathrm{E}$ & & & & & & & & & & & & & & & & $* * * * *$ & $* * * * *$ & $* * * * * *$ & $* * * * *$ & $* * * * * *$ \\
\hline $4 \mathrm{~A}$ & & & & & & & & & & & & & & & & & $* * * * * *$ & & $* * * * *$ & $* * * * *$ \\
\hline $4 \mathrm{~B}$ & & & & & & & & & & & & & & & & & & $* * * * *$ & $* * * * *$ & $* * * * *$ \\
\hline $4 \mathrm{C}$ & & & & & & & & & & & & & & & & & & & $* * * * * *$ & $* * * * * *$ \\
\hline $4 \mathrm{D}$ & & & & & & & & & & & & & & & & & & & & $* * * * * *$ \\
\hline $4 \mathrm{E}$ & & & & & & & & & & & & & & & & & & & & \\
\hline
\end{tabular}




\title{
4. Conclusions
}

The vacuum-packed yellowfin tuna loins are at the top of the risk as regards the presence of histamine in fish products. Moreover, the EU has banned CO treatment in fish: in the treated products, the colour may mask the deterioration associated with potential risk of scombroid syndrome [13] and "suspect" samples for CO should also be sampled for histamine. The determination of the colorimetric parameters was intended to highlight any correlations between high levels of histamine and a possible use of $\mathrm{CO}$ through a quick and easy-to-use colorimetric test. Although many of the yellowfin tuna samples included in our study showed a bright red cherry colour with significant $(p<0.05)$ differences, even in presence of "suspicious" staining, the comparison between the colorimetric parameters and the results of the quantitative determination of the histamine level, did not show any substantial correlation. Marketing practices may significantly influence the levels of histamine in yellowfin tuna: the highest levels were observed in samples collected from small fishmongers and local markets, where were displayed for more than $8 \mathrm{~h}$ at temperatures $>5^{\circ} \mathrm{C}$ considerably compromising the safety and quality of yellowfin tuna [57]. According to previous studies [34] which reported a good overlap of the results obtained from HistaSure ${ }^{\mathrm{TM}}$ Fish Rapid Test (LDN) with HPLC results, this study showed an agreement of $85 \%$ between the results obtained by rapid screening methods and those obtained by the official LC-MS/MS method. However, $10 \%$ of false negative results and $5 \%$ of false positive results obtained in this study highlighted that lateral flow tests must be reinforced by official methods if doubtful or positive results are achieved [50].

\begin{abstract}
Author Contributions: Conceptualization, G.L.P., D.M. and G.C.; methodology, G.L.P., L.C. and A.P. software, D.M.; validation, D.M., A.G.M. and G.C.; formal analysis, G.L.P., L.C. and A.P.; investigation, D.M.; resources, D.M.; data curation, D.M. and G.C.; writing-original draft preparation, G.L.P., D.M. and G.C.; writing-review and editing, G.L.P., D.M. and G.C.; visualization, A.G.M.; supervision, D.M. and G.C.; project administration, D.M.; funding acquisition, D.M. All authors have read and agreed to the published version of the manuscript.
\end{abstract}

Funding: This research And The APC were funded by FAR2019 (Fondo di Ateneo per la Ricerca 2019) and FAR2020 (Fondo di Ateneo per la Ricerca 2020) UNISS.

Institutional Review Board Statement: Not applicable.

Informed Consent Statement: Not applicable.

Data Availability Statement: Not applicable.

Conflicts of Interest: The authors declare no conflict of interest.

\section{References}

1. FAO (Food and Agriculture Organization of the United Nations). The State of World Fisheries and Aquaculture 2020; Sustainability in Action; FAO: Rome, Italy, 2020. [CrossRef]

2. Asensio, L.; Montero, A. Analysis of fresh fish labelling in Spanish fish retail shops. Food Control 2008, 19, 795-799. [CrossRef]

3. Ministero delle Politiche Agricole, Alimentari e Forestali (MiPAAF). The State of Italian Fisheries and Aquaculture; Cataudella, S., Spagnolo, M., Eds.; Ministero delle Politiche Agricole, Alimentari e Forestali (MiPAAF): Rome, Italy, 2011. Available online: https:/ / www.politicheagricole.it/flex/cm/pages/ServeBLOB.php/L/IT/IDPagina/6412 (accessed on 4 December 2021).

4. Maralit, B.A.; Aguila, R.D.; Ventolero, M.F.H.; Perez, S.K.L.; Willette, D.A.; Santos, M.D. Detection of mislabeled commercial fishery by-products in the Philippines using DNA barcodes and its implications to food traceability and safety. Food Control 2013, 33, 119-125. [CrossRef]

5. Sterling, B.; Chiasson, M.A. Enhancing Seafood Traceability Issues Brief; Global Food Traceability Center: Chicago, IL, USA, 2014. [CrossRef]

6. Pramod, G.; Nakamura, K.; Pitcher, T.J.; Delagran, L. Estimates of illegal and unreported fish in seafood imports to the USA. Mar. Policy 2014, 48, 102-113. [CrossRef]

7. Aung, M.M.; Chang, Y.S. Traceability in a food supply chain: Safety and quality perspectives. Food Control 2014, 39, 172-184. [CrossRef]

8. Parreño-Marchante, A.; Alvarez-Melcon, A.; Trebar, M.; Filippin, P. Advanced traceability system in aquaculture supply chain. J. Food Eng. 2014, 122, 99-109. [CrossRef] 
9. Leal, M.C.; Pimentel, T.; Ricardo, F.; Rosa, R.; Calado, R. Seafood traceability: Current needs, available tools, and biotechnological challenges for origin certification. Trends Biotechnol. 2015, 33, 331-336. [CrossRef]

10. D'Amico, P.; Armani, A.; Castigliego, L.; Sheng, G.; Gianfaldoni, D.; Guidi, A. Seafood traceability issues in Chinese food business activities in the light of the European provisions. Food Control 2014, 35, 7-13. [CrossRef]

11. Esposito, G.; Meloni, D. A case-study on compliance to the EU new requirements for the labelling of fisheries and aquaculture products reveals difficulties in implementing Regulation (EU) n.1379/2013 in some large-scale retail stores in Sardinia (Italy). Reg. Stud. Mar. Sci. 2017, 9, 56-61. [CrossRef]

12. Al-Abdessalaam, T.J.S. Marine Species of the of the Sultanate of Oman: An Identification Guide; Ministry of Agriculture and Fisheries, Publication No. 46/95; Muscat Printing Press: Muscat, Oman, 1995; p. 412.

13. Marrone, R.; Mascolo, C.; Palma, G.; Smaldone, G.; Girasole, M.; Anastasio, A. Carbon monoxide residues in vacuum-packed yellowfin tuna loins (Thunnus Albacares). Ital. J. Food Saf. 2015, 4, 142-144. [CrossRef]

14. Europol. How the illegal Bluefin tuna market made over EUR 12 million a year selling fish in Spain. 2018. Available online: https:/ / www.europol.europa.eu/newsroom/news/how-illegal-bluefin-tuna-market-made-over-eur-12-million-yearselling-fish-in-spain (accessed on 3 December 2021).

15. Evangelista, W.P.; Silva, T.M.; Guidi, L.R.; Tette, P.A.S.; Byrro, R.M.D.; Santiago-Silva, P.; Fernandes, C.; Gloria, M.B.A. Quality assurance of histamine analysis in fresh and canned fish. Food Chem. 2016, 211, 100-106. [CrossRef]

16. Altieri, I.; Semeraro, A.; Scalise, F.; Calderari, I.; Stacchini, P. European official control of food: Determination of histamine in fish products by a HPLC- UV-DAD method. Food Chem. 2016, 211, 694-699. [CrossRef]

17. Nei, D.; Nakamura, N.; Ishihara, K.; Kimura, M.; Satomi, M. A rapid screening of histamine concentration in fish fillet by direct analysis in real time mass spectrometry (DART-MS). Food Control 2017, 75, 181-186. [CrossRef]

18. Silva, T.M.; Sabaini, P.S.; Evangelista, W.P.; Gloria, M.B.A. Occurrence of histamine in Brazilian fresh and canned tuna. Food Control 2011, 22, 323-327. [CrossRef]

19. Colombo, F.M.; Cattaneo, P.; Confalonieri, E.; Bernardi, C. Histamine food poisonings: A systematic review and meta-analysis. Crit. Rev. Food Sci. Nutr. 2018, 58, 1131-1151. [CrossRef] [PubMed]

20. European Food Safety Authority (EFSA). Assessment of the Incidents of Histamine Intoxication in Some EU Countries. 2017. Available online: https:/ / www.efsa.europa.eu/en/supporting/pub/en-1301 (accessed on 3 December 2021).

21. Ministero della Salute, Direzione Generale per l'Igiene e la Sicurezza degli Alimenti e la Nutrizione, Ufficio 8. RASFF Rapid Alert System for Food and Feed-Sistema di Allerta Rapido per Alimenti e Mangimi. Relazione Annuale 2020; Italian Health Ministry: Rome, Italy, 2021. Available online: https:/ / www.salute.gov.it/imgs/C_17_pubblicazioni_3100_allegato.pdf (accessed on 5 December 2021).

22. European Commission. Commission Regulation (EC) No 2073/2005 of 15 November 2005 on microbiological criteria for foodstuffs. Off. J. Eur. Union 2005, L 338/1-26.

23. Nadeem, M.; Naveed, T.; Rehman, F.; Xu, Z. Determination of histamine in fish without derivatization by indirect reverse phase-HPLC method. Microchem. J. 2019, 144, 209-214. [CrossRef]

24. Esatbeyoglu, T.; Ehmer, A.; Chaize, D.; Rimbach, G. Quantitative Determination of Spermidine in 50 German Cheese Samples on a Core-Shell Column by High-Performance Liquid Chromatography with a Photodiode Array Detector Using a Fully Validated Method. J. Agric. Food Chem. 2016, 64, 2105-2111. [CrossRef]

25. Tibor, J.; Davor, V.; Jasenka, G.; Gajdos, K.; Lara, M.; Sanja, V.; Mile, I. Determination of histamine in fish by Surface Enhanced Raman Spectroscopy using silver colloid SERS substrates. Food Chem. 2017, 224, 48-54.

26. Khan, S.; Carneiro, L.S.A.; Vianna, M.S.; Romani, E.C.; Aucelio, R.Q. Determination of histamine in tuna fish by photoluminescence sensing using thioglycolicacid modified CdTe quantum dots and cationic solid phase extraction. J. Lumin. 2017, 182, 71-78. [CrossRef]

27. Lili, H.; Zhongqi, X.; Takeshi, H.K.; Li, S. Simultaneous determination of aliphatic, aromatic and heterocyclic biogenic amines without derivatization by capillary electrophoresis and application in beer analysis. J. Chromatogr. A 2017, 1482, 109-114.

28. Surya, T.; Sivaraman, B.; Alamelu, V.; Priyatharshini, A.; Arisekar, U.; Sundhar, S. Rapid Methods for Histamine Detection in Fishery Products. Int. J. Curr. Microbiol. Appl. Sci. 2019, 8, 2035-2046. [CrossRef]

29. Bajpai, V.K.; Oh, C.; Khan, I.; Haldorai, Y.; Gandhi, S.; Lee, H.; Song, X.; Kim, M.; Upadhyay, A.; Chen, L.; et al. Fluorescent immunoliposomal nanovesicles for rapid multi-well immuno-biosensing of histamine in fish samples. Chemosphere 2020, 243, 125404. [CrossRef] [PubMed]

30. Papageorgiou, M.; Lambropoulou, D.; Morrison, C.; Kłodzinska, E.; Namieśnik, J.; Płotka-Wasylka, J. Literature update of analytical methods for biogenic amines determination in food and beverages. Trends Anal. Chem. 2018, 98, 128-142. [CrossRef]

31. Stojanović, Z.; Kos, J. 2-Detection of Metabolites of Microbial Origin in Beverages with Harmful Effect on Human HealthBiogenic Amines and Mycotoxins. Saf. Issues Beverage Prod. 2020, 18, 39-77.

32. Anithaa, A.C.; Mayil Vealan, S.B.; Veerapandi, G.; Sekar, C. Highly efcient non-enzymatic electrochemical determination of histamine based on tungsten trioxide nanoparticles for evaluation of food quality. J. Appl. Electrochem. 2021, 51, 1741-1753. [CrossRef]

33. Rogers, P.L.; Staruszkiewicz, W.F. Histamine Test Kit Comparison. J. Aquat. Food Prod. Technol. 2000, 9, 5-17. [CrossRef]

34. Köse, S.; Kaklikkaya, N.; Koral, S.; Tufan, B.; Buruk, K.C.; Aydın, F. Commercial test kits and the determination of histamine in traditional (ethnic) fish products-evaluation against an EU accepted HPLC method. Food Chem. 2011, 125, 1490-1497. [CrossRef] 
35. Crobu, L.; Mudadu, A.G.; Melillo, R.; Pais, G.L.; Meloni, D. Qualitative determination of histamine in canned yellowfin tuna (Thunnus albacares) marketed in Sardinia (Italy) by rapid screening methods. Ital. J. Food Saf. 2021, 10, 9379. [CrossRef]

36. 36. European Council. Council Regulation (EEC) No 1536/92 of 9 June 1992 laying down common marketing standards for preserved tuna and bonito. Off. J. Eur. Communities 1992, L163/1-4.

37. European Union (EU). Regulation No 1379/2013 of the European Parliament and of the Council of 11 December 2013 on the common organisation of the markets in fishery and aquaculture products, amending Council Regulations (EC) No 1184/2006 and (EC) No 1224/2009 and repealing Council Regulation (EC) No 104/2000. Off. J. Eur. Union 2013, L 354/1-21.

38. Commission Internationale de l'Eclairage. Colorimetry_Part 4: CIE $1976 L^{*} a^{*} b^{*}$ Colour Spaces; Publication CIE: Vienna, Austria, 2008.

39. Meloni, D.; Spina, A.; Satta, G.; Chessa, V. A rapid colorimetric method reveals fraudulent substitutions in sea urchin roe marketed in Sardinia (Italy). Foods 2016, 5, 47. [CrossRef]

40. Instructions for use HistaSure ${ }^{\mathrm{TM}}$ Fish Rapid Test. Available online: https://ldn.de/wp-content/uploads/fc-1-3200-en-v13.0_wz. pdf (accessed on 5 December 2021).

41. AOAC. AOAC Official Method 937.07 Fish and Marine Products-Treatment and Preparation of Sample-Procedure. In Official Methods of Analysis of AOAC International, 17th ed.; AOAC International: Gaithersburg, MD, USA, 2000; Section, 35.1.01.

42. Interactive Statisic-Analysis of Variance from Summary Data. Available online: https://statpages.info/anova1sm.html (accessed on 2 December 2021).

43. Koohdar, V.A.; Razavilar, V.; Motalebi, A.A.; Mosakhani, F.; Valinassab, T. Isolation and Identification of Histamine-forming bacteria in frozen Skipjack tuna (Katsuwonus pelamis). Iran. J. Fish Sci. 2010, 10, 678-688.

44. Mercogliano, R.; Santonicola, S. Scombroid fish poisoning: Factors influencing the production of histamine in tuna supply chain. A review. LWT Food Sci. Technol. 2019, 114, 108374. [CrossRef]

45. Yoshinaga, D.H.; Frank, H.A. Histamine-Producing bacteria in decomposing skipjack tuna (Katsuwonus pelamis). Appl. Environ. Microbiol. 1982, 44, 447-452. [CrossRef]

46. Meloni, D.; Piras, P.; Mazzette, R. Mislabelling and species substitution in fishery products retailed in Sardinia (Italy), 2009-2014. Ital. J. Food Saf. 2015, 4, 199-203. [CrossRef]

47. Meloni, D. Labelling and marketing of bivalve and gastropod molluscs retailed in Sardinia, Italy between 2009 and 2013. Ital. J. Food Saf. 2015, 4, 104-106. [CrossRef]

48. Dambrosio, A.; Anaclerio, D.; Quinto, M.; Centoducati, G.; Errico, L.; Girolamo, L.G.; Normanno, G. Indagine sull'applicazione delle norme sull'etichettatura di prodotti ittici commercializzati al dettaglio in Puglia: Risvolti normativi e igienico-sanitari. Ind. Aliment. 2012, 51, 40-46.

49. Brom, F.W.A. Food, consumer concerns, and trust: Food ethics for a globalizing market. J. Agric. Environ. Ethics 2000, 12, 127-139. [CrossRef]

50. EUFIC (European Food Information Council). Pan-European Consumer Research on In-Store Observation, Understanding \& Use of Nutrition Information on Food Labels, Combined with Assessing Nutrition Knowledge; EUFIC Forum N ${ }^{\circ} 4.2009$. Available online: https://www.eufic.org/en/images/uploads/files/Pan-EU_executive_summary_FINAL.pdf (accessed on 7 December 2021).

51. Bonsmann, S.; Fernández-Celemín, L.; Grunert, K.G. Food labelling to advance better education for life. Eur. J. Clin. Nutr. 2010, 64, S14-S19. [CrossRef]

52. Beke, M.; Blomeyer, R. Illegal, Unreported and Unregulated Fishing: Sanctions in the EU. Study. European Parliament. Directorate-General for Internal Policies. Policy Department B: Structural and Cohesion Policies. Fisheries. 2014. Available online: https:/ / www.europarl.europa.eu/RegData/etudes/STUD/2014/529069/IPOL_STU(2014)529069_EN.pdf (accessed on 7 December 2021).

53. Petrossian, G.A. Preventing illegal, unreported and unregulated (IUU) fishing: A situational approach. Biol. Cons. 2015, 189, 39-48. [CrossRef]

54. Visciano, P.; Schirone, M.; Paparella, A. An overview of histamine and other biogenic amines in fish and fish products. Foods 2020, 9, 1795. [CrossRef] [PubMed]

55. Tao, Z.; Sato, M.; Zhang, H.; Yamaguchi, T.; Nakano, T. A survey of histamine content in seafood sold in markets of nine countries. Food Control 2011, 22, 430-432. [CrossRef]

56. Bilgin, B.; Gençcelep, H. Determination of biogenic amines in fish products. Food Sci. Biotechnol. 2015, 24, 1907-1913. [CrossRef]

57. Chummun, S.; Neetoo, H. A Study on the Relationship between Microbial Growth, Histamine Development and Organoleptic Changes in Retailed Fresh Sprangled Emperor and Big Eye Tuna. J. Food Chem. Nanotechnol. 2016, 2, 6-13. [CrossRef] 